\title{
Pressure effects on the magnetic properties of FeCuZr studied by x-ray magnetic circular dichroism: Evidence of weakening of ferromagnetism in FeCuZr alloys
}

\author{
A. Martínez, ${ }^{1}$ J. J. Romero, ${ }^{2}$ F. Bartolomé, ${ }^{3}$ L. M. García, ${ }^{3}$ F. Baudelet,${ }^{4}$ A. Hernando, ${ }^{1}$ \\ and P. Crespo ${ }^{1}$ \\ ${ }^{1}$ Instituto de Magnetismo Aplicado (UCM-ADIF-CSIC), P.O. Box 155, 28230 Las Rozas, Madrid, Spain \\ ${ }^{2}$ Instituto de Cerámica y Vidrio (CSIC) C/Kelsen, 28049 Madrid, Spain \\ ${ }^{3}$ Instituto de Ciencia de Materiales de Aragón and Departamento de Física de la Materia Condensada \\ (CSIC-Universidad de Zaragoza), Pedro Cerbuna 12, 50009 Zaragoza, Spain \\ ${ }^{4}$ SOLEIL, L'orme des Merisiers, Saint Aubin B.P. 48, 91192 Gif-sur-Yvette cedex, France
}

(Received 20 April 2012; accepted 23 June 2012; published online 12 July 2012)

\begin{abstract}
The room temperature changes of the magnetic behavior under pressure of an invar alloy of nominal composition $\left(\mathrm{Fe}_{0.5} \mathrm{Cu}_{0.5}\right)_{87} \mathrm{Zr}_{13}$ (at. \%) has been studied by K-edge $\mathrm{x}$-ray magnetic circular dichroism (XMCD) and x-ray absorption near edge spectroscopy (XANES). The Curie temperature, as determined from low field magnetization measurements, is $(255 \pm 15) \mathrm{K}$. However $\mathrm{XMCD}$ shows a non negligible signal above this temperature. In addition, the XMCD signal decreases upon increasing pressure indicating a pressure-induced collapse of the magnetic moment. These results evidence the itinerant character of FeCuZr alloys as well as the occurrence of magnetovolume effects characterized by a strong dependence of the $3 \mathrm{~d}$ band on the $\mathrm{Fe}-\mathrm{Fe}$ nearest neighbor distances. (C) 2012 American Institute of Physics. [http://dx.doi.org/10.1063/1.4735300]
\end{abstract}

Magnetism plays a fundamental role in stabilizing the ambient pressure phases of $3 \mathrm{~d}$ transition metals. In particular, Fe exhibit a body centered cubic phase (BCC) instead of the hexagonal close packed (HCP) structure predicted without spin polarization. ${ }^{1}$ Under compression, the ferromagnetic character of BCC-Fe disappears at around $16 \mathrm{GPa}$, a behavior that is connected with the structural transition from a BCC structure to a HCP. ${ }^{2}$ This transition is accompanied by the disappearance of the Fe magnetic moment, suggesting that HCP-Fe is non magnetic. The BCC-Fe phase is ferromagnetic with a Curie temperature (Tc) of $1043 \mathrm{~K}$ and is the stable phase up to $1183 \mathrm{~K}$. At this temperature, there is a structural phase transformation into the face centered cubic (FCC) structure, the so-called gamma-Fe phase, being not magnetic at this temperature range. Pure FCC-Fe is only stable at high temperatures, where no spontaneous magnetic ordering is observed. However, several theoretical works have shown that the magnetism of FCC-Fe is strongly dependent on interatomic distances, exhibiting a wide and rich phenomelogy, ranging from non-collinear, helical, non magnetic, antiferromagnetic, as well as ferromagnetic ordering of Fe moments. ${ }^{3}$

The dependence of magnetic structures on the interatomic distances suggests that FCC-Fe and FCC-Fe alloys are potential candidates for exhibiting magnetovolume effects. Strong magnetovolume effects, such high volume magnetostriction pressure effects on magnetization, and Curie temperature as well as invar and anti-invar behavior have been reported not only in $\mathrm{Fe}$ alloys with FCC structure, such as $\mathrm{FeNi}^{4}{ }^{4} \mathrm{FePt},{ }^{5} \mathrm{FeCu},{ }^{6-8}$ but also in $\mathrm{Fe}$ based amorphous alloys. ${ }^{9,10}$ Such effects are likely caused by the dependence of the band width on the atomic distances, in consequence the magnetic structure changes upon modification of the lattice dimensions. ${ }^{11}$
In a previous work, we reported on the anomalous temperature dependence of the thermoremanence (TRM) exhibited by amorphous $\mathrm{FeCuZr}$ alloys and the link of such behavior with the evolution of the Fe-Fe nearest neighbor (NN) distances with temperature. It was observed that the TRM decreases upon increasing the temperature up to the $\mathrm{Cu}-$ rie temperature $(\mathrm{Tc})$ of the alloy $(255 \pm 15) \mathrm{K} .{ }^{12}$ At this temperature, the TRM becomes negligible whereas further increase in temperature promotes a spontaneous, i.e., in absence of an applied magnetic field, recovering of the magnetization. As mentioned, it was shown that this behavior is closely correlated with the temperature dependence of Fe-Fe $\mathrm{NN}$ distances determined from extended $\mathrm{x}$-ray absorption fine structure (EXAFS) studies. The evolution of Fe-Fe NN distances with temperature show two different regimes: (i) below the Curie temperature the alloy shows an invar behavior with an almost no thermal dependence of the Fe-Fe NN distance; (ii) above the Curie temperature normal thermal expansion takes place and, in consequence, the $\mathrm{Fe}-\mathrm{Fe} \mathrm{NN}$ distances increases. In the invar regime, the decrease of the magnetization causes the shrinking of the lattice compensating for the contribution arising from the anharmonicity of the lattice vibrations, whereas the spontaneous increase of the magnetization is directly related with the increase of the interatomic distances. The ferromagnetic interaction among $\mathrm{Fe}$ atoms is enhanced upon increasing $\mathrm{Fe}-\mathrm{Fe} \mathrm{NN}$ distances and, in consequence, for each state of dilation there is an associated Tc.

Thus, two questions arise (i) is the recovering of the magnetization above the Tc of the alloy arising from an enhancement of the ferromagnetic interactions due to thermal lattice expansion? (ii) If it so, this behavior should arise from changes in the band structure due to volume expansion, thus ferromagnetism should disappear by applying a high enough pressure. 
For clarifying whether FeCuZr exhibit such strong correlation among the magnetic structure and $\mathrm{Fe}-\mathrm{Fe}$ NN distances, XANES, and XMCD measurements were carried out under pressure at room temperature.

Powders of composition $\left(\mathrm{Fe}_{0.5} \mathrm{Cu}_{0.5}\right)_{87} \mathrm{Zr}_{13}$ (at. \%) were prepared by high energy ball milling in a planetary mill with hardened steel vials and balls. Powders of metallic iron, copper, and zirconium, with $99.9 \%$ of purity, were the starting materials. For avoiding the oxidation of the sample, the vial was sealed in an oxygen-free atmosphere. The sample was milled $120 \mathrm{~h}$ with a cycle of $50 \mathrm{~s}$ work and $10 \mathrm{~s}$ stop in order to avoid the excessive heat that could difficult the alloying of the materials. X-ray diffraction pattern, obtaining with CoKa radiation, exhibit the typical broad halo of amorphous alloys (see Fig. 1). The amorphous state is also confirmed by transmission electron microscopy (TEM). The diffraction pattern shows the typical rings and absence of diffraction spots expected for amorphous materials, as can be also observed in Fig. 1.

XANES and XMCD measurements were carried out at the optique dispersive EXAFS (ODE) beamline at SOLEIL in transmission geometry. For performing the high pressure measurements, the powders were loaded in a diamond-anvil cell (DAC) using mineral oil as pressure medium. The pressure was controlled by measuring the luminescence properties of a ruby crystal located near to the sample. ${ }^{13}$

Since diamond is transparent for $\mathrm{x}$-ray energies above $5 \mathrm{keV}$ and the measurements are done in transmission geometry, the measurements were conducted at $\mathrm{Fe}-\mathrm{K}$ edge $(7112 \mathrm{eV})$, that corresponds to transitions to the $4 p$ conduction band. The spectra were recorded under by applying pressures up to $24.6 \mathrm{GPa}$ at room temperature. The incident radiation was circularly polarized and, at each pressure, two spectra were collected under two different polarizing magnetic fields, $\mu_{0} \mathrm{H}= \pm 1,3 \mathrm{~T}$. The magnetic field was applied along the x-ray direction by means of an electromagnet. The XMCD signal is obtained by subtracting the XANES spectra obtained in the two different magnetic states of the sample, under the same conditions of temperature and pressure.

Although $\mathrm{Fe}-\mathrm{K}$ edge does not allow probing directly the $3 \mathrm{~d}$ orbitals but the extended $4 \mathrm{p}$ bands, it has been previously reported that the XMCD signal at K-edge for 3d metal is related with the spin polarization of $4 p$ band. Since changes in the $3 \mathrm{~d}$ magnetic moment influences the exchange splitting

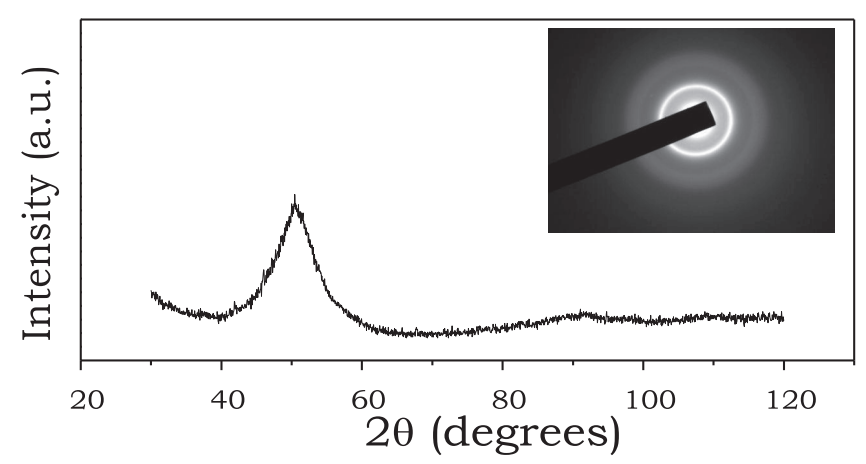

FIG. 1. X-ray diffraction pattern of as-milled $\left(\mathrm{Fe}_{0.5} \mathrm{Cu}_{0.5}\right)_{87} \mathrm{Zr}_{13}$ (at. \%) alloy. The inset shows the electron diffraction pattern, evidencing the amorphous nature of the alloy.

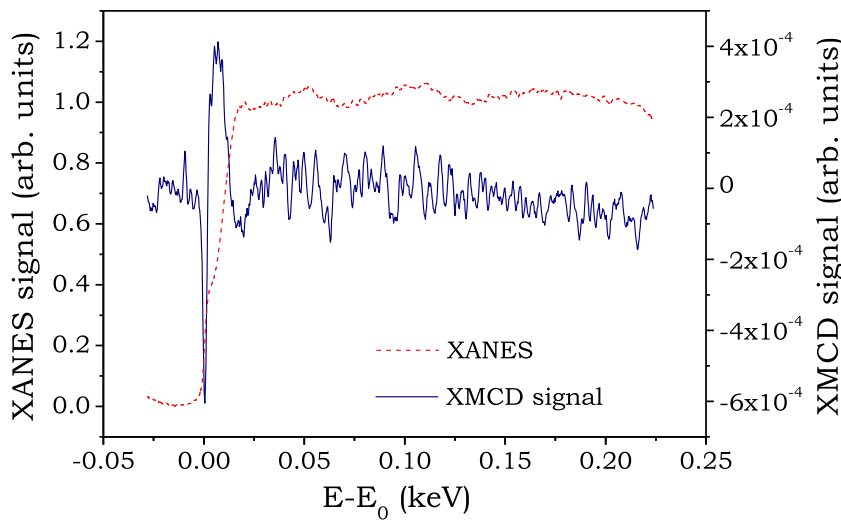

FIG. 2. XANES spectrum (dashed line) and XMCD signal (solid line) measured at room temperature and under no applied pressure. The energy is normalized to Fe-K edge.

of the $4 p$ band, the K-edge signal can be used for studying the $3 \mathrm{~d}$ magnetic moment. ${ }^{14}$

XANES spectrum and XMCD signal of amorphous $\left(\mathrm{Fe}_{0.5} \mathrm{Cu}_{0.5}\right)_{87} \mathrm{Zr}_{13}$ (at. \%) at room temperature (RT) and at ambient pressure are shown in Fig. 2. The XANES spectrum is in well agreement with previous measurements performed on the same material. ${ }^{12,15}$ The average $\mathrm{NN}$ Fe-Fe distance has been estimated as to be about $2.37 \AA$.

It should be remarked that the XMCD signal is not negligible at room temperature, as Fig. 2 clearly shows. This result indicates that the alloy exhibit magnetic ordering of the iron atoms at room temperature, in good agreement with previously reported magnetic data. ${ }^{12}$ Since the Curie temperature of the alloy is around $255 \mathrm{~K}$, as determined from low field magnetic measurements, ${ }^{12}$ the experimental observation obtained from XMCD data confirms that the reported lattice thermal dilation that takes place above Tc allows recovering the ferromagnetic interaction among Fe atoms. By increasing the Fe-Fe NN distances, the alloy exhibit higher Curie temperatures. Thus, this indicates that for each state of dilation there is an associated Tc.

The above results indicate that the magnetic structure of the alloy is, in consequence, highly dependent on $\mathrm{Fe}-\mathrm{Fe} \mathrm{NN}$ distances. This feature is a characteristic of alloys that exhibit strong magnetovolume effects. In consequence, a decrease of the ferromagnetic ordering should be expected by applying a high enough pressure. Figure 3 shows the

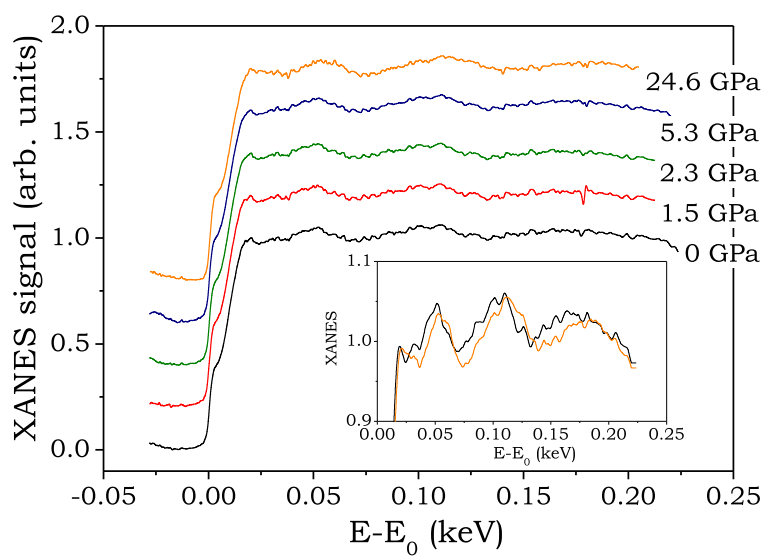

FIG. 3. Room temperature Fe K-edge XANES spectra as a function of applied pressure. 


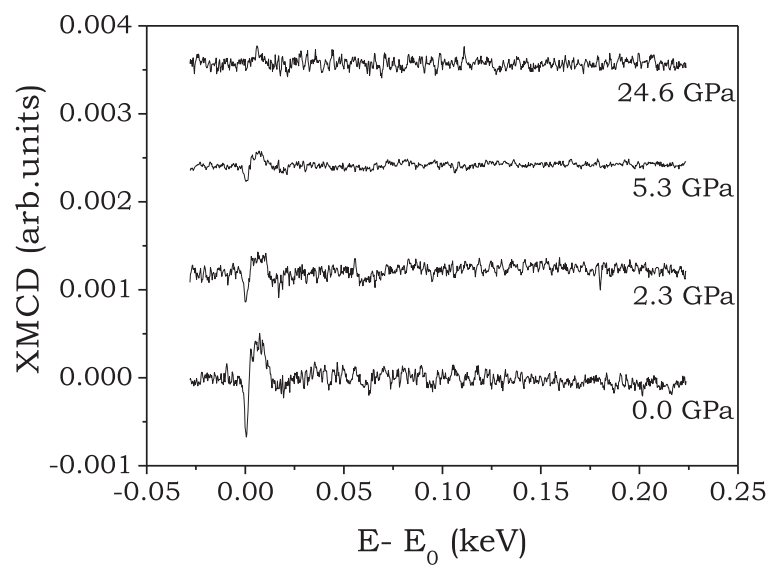

FIG. 4. Evolution of room temperature XMCD signal with applied pressure. The energy is normalized at Fe K-edge.

XANES spectra measured at Fe-K edge under increasing pressures, up to $24 \mathrm{GPa}$. Although a shift of the maxima in the near EXAFS region is appreciable as pressure increases (see inset in Fig. 3), the k-range explored in the XMCD experiment is not sufficient to quantitatively determine the variation in $\mathrm{Fe}-\mathrm{Fe}$ distances.

Figure 4 shows the pressure induced changes in the Fe-K edge XMCD at room temperature whereas Fig. 5 summarizes the $\mathrm{XMCD}$ peak to peak signal $\left(\mathrm{XMCD}_{\mathrm{p}-\mathrm{p}}\right)$ as a function of pressure. XMCD peak to peak signal has been estimated from difference between the minimum and the maximum values of the XMCD signal around the Fe K-edge. It is clearly observed that the XMCD signal initially decreases sharply with pressure, a decrease of around $29.6 \%$ for pressures between $0 \mathrm{GPa}$ and 5,6 GPa, whereas for pressures above $5.6 \mathrm{GPa}$ the decrease observed up to $24.6 \mathrm{GPa}$ is much smaller, being around 5.9\%.

The decrease of the dichroism signal indicates a loss of long-range ferromagnetic order in the alloy.

Previous works have reported a decrease of the $\mathrm{Fe}$ $\mathrm{K}$-edge XMCD signal in BCC Fe. By increasing the pressure, it is observed that the XMCD signal decreases slightly up to pressures of $12 \mathrm{GPa}$. Further increase in pressure promotes the BCC-HCP transition (12-16 GPa) that is accompanied by a sharp decrease of the XMCD signal. The XMCD signal is

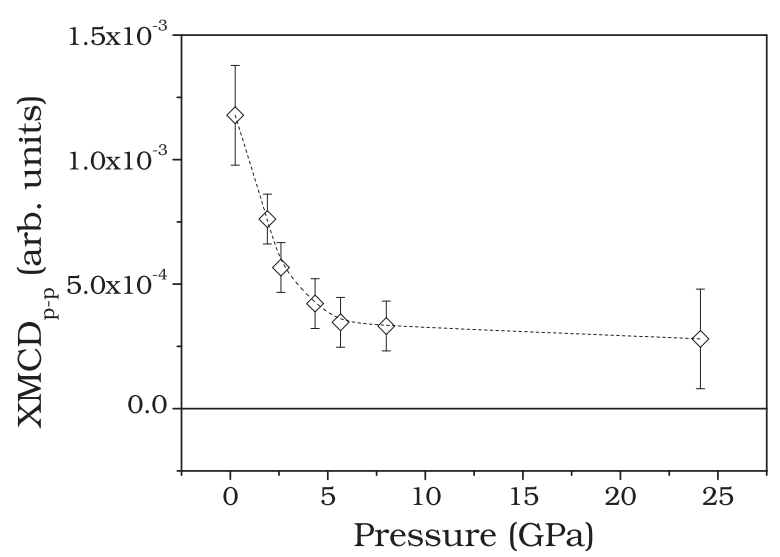

FIG. 5. Evolution of the XMCD peak to peak signal with pressure. XMCD peak to peak signal has been estimated form the distance between the minimum and the maximum in the XMCD signal spectra. almost null for pressures above $23 \mathrm{GPa}$ due to the full transformation into the HCP-Fe phase. ${ }^{2}$ One can argue whether the strong decrease observed in $\mathrm{FeCuZr}$ at low pressures could be related with a pressure-induced structural transition in the alloy similar as that observed in BCC Fe. However, this seems not to be the case since the XANES spectra does not change in shape upon increasing pressure (see Fig. 6).

Thus, the observed decrease in the XMCD signal and, in consequence, the corresponding decrease in the Fe magnetic moment should be related to the pressure-induced compression of the alloy that sharply modifies the magnetic behavior. As previously mentioned, in these alloys it has been observed a spontaneous enhancement of the magnetization upon increasing temperatures that is directly correlated with a thermal increase of the Fe-Fe nearest neighbor distances determined from EXAFS measurements. In the present work, the reversal effect is reported. The pressure increases the overlap between $3 \mathrm{~d}-4 \mathrm{p}$ band that promotes an increase in the $3 \mathrm{~d}$ bandwidth resulting a in reduction in the magnetic moment of $\mathrm{Fe}$ atoms.

According to the Stoner criterion, an itinerant ferromagnet would become spontaneously ferromagnetic when $\operatorname{I.} g\left(E_{\mathrm{F}}\right)>1$, where $I$ is the Stoner parameter, which is a measure of the strength of the exchange interaction, and $g\left(E_{\mathrm{F}}\right)$, is the density of states at the Fermi level. Since the Stoner parameter does not depend on the volume, the dependence of the Stoner criterion on the Fe-Fe NN distances arises from the density of states at the Fermi level. It is well established that an increase in the $3 \mathrm{~d}$ band width would lead to a decrease in the density of states at the Fermi level and, in consequence, to a weakening of ferromagnetic interactions. It has been shown that the magnetic structure of $\mathrm{FeCu}$ based alloys exhibit a strong dependence of the interatomic distances. Equiatomic fcc-FeCu alloys exhibit a positive deviation from Vegard's law, i.e., the lattice is expanded with respect to that of fcc-Cu. In fact, ferromagnetism in equiatomic fcc$\mathrm{FeCu}$ alloys has been attributed to magnetovolume effect. The incorporation of Fe into the $\mathrm{Cu}$ lattice allows stabilizing a high volume/high spin state of $\mathrm{Fe}$ that would account for the ferromagnetic behavior in the binary alloy, a behavior that fcc-Fe and fcc-Cu do not exhibit at their ground state.

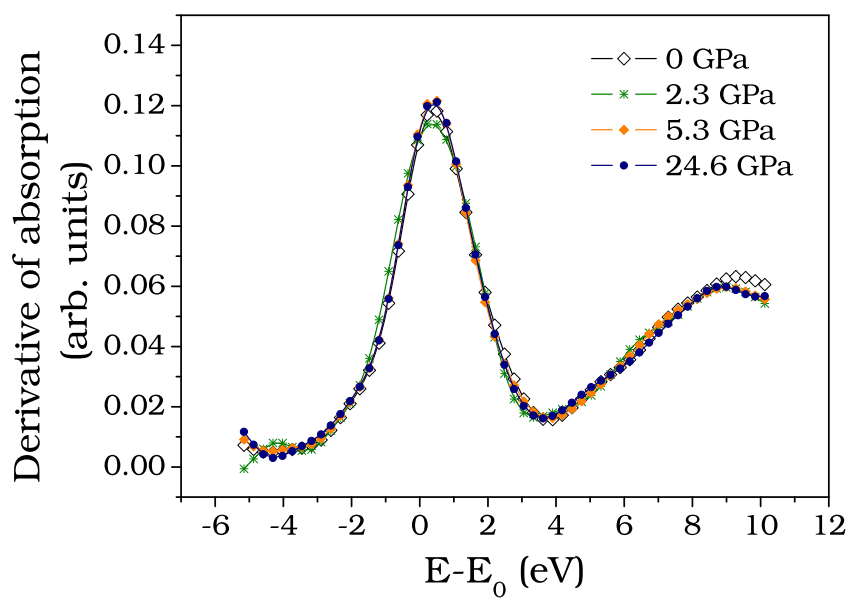

FIG. 6. Derivative of the absorption with respect to energy for different applied pressures. Notice that the shape does not change upon increasing pressure. 
The addition of $\mathrm{Zr}$ decreases the interatomic distances. The lattice parameter, determined form XRD measurements of equiatomic disordered fcc- $\mathrm{Fe}_{50} \mathrm{Cu}_{50}$ is $\mathrm{a}=0.3641 \mathrm{~nm}$, thus the average distance to nearest neighbor is $\mathrm{a}=0.2574 \mathrm{~nm}$, whereas in the case of the $\left(\mathrm{Fe}_{0.5} \mathrm{Cu}_{0.5}\right)_{87} \mathrm{Zr}_{13}$ (at. \%) alloy, the average $\mathrm{Fe}-\mathrm{Fe} \mathrm{NN}$ distance determined from EXAFS is around $\mathrm{a}=0.237 \mathrm{~nm}$. When the magnetic behavior of both compositions is compared, it is observed that the Curie temperature decreases from $550 \mathrm{~K}$ to $255 \mathrm{~K}$, for $\mathrm{x}=00$ and $\mathrm{x}=13$ respectively, whereas the atomic magnetic moment also decreases from $2.14 \mu_{\mathrm{B}}$ to $1.75 \mu_{\mathrm{B}}$, being $\mu_{\mathrm{B}}$ the Bohr magneton. Although both alloys are not directly comparable, since $\mathrm{Fe}_{50} \mathrm{Cu}_{50}$ is a nanocrystalline solid solution with a FCC structure characterized by different nonequivalent $\mathrm{Fe}$ positions, as Mössbauer spectroscopy points out ${ }^{16}$ whereas the $\left(\mathrm{Fe}_{0.5} \mathrm{Cu}_{0.5}\right)_{87} \mathrm{Zr}_{13}$ (at.\%) composition is an amorphous alloy, it seems that the addition of $\mathrm{Zr}$ decreases $\mathrm{Fe}-\mathrm{Fe} \mathrm{NN}$ distances that affect the magnetic behavior. In particular, it seems that a decrease of $\mathrm{Fe}-\mathrm{Fe} \mathrm{NN}$ distances promotes a softening of the ferromagnetic interaction among Fe atoms. By applying a high enough pressure, $\mathrm{XMCD}$ becomes almost negligible, a clear fingerprint of a weakening of the ferromagnetic interactions.

In summary, we have described XMCD measurements performed at $\mathrm{Fe} \mathrm{K}$-edge and under pressure on an invar alloy $\left(\mathrm{Fe}_{0.5} \mathrm{Cu}_{0.5}\right)_{87} \mathrm{Zr}_{13}$ (at. \%). The XMCD signal decreases under compression indicating that the magnetic moment decreases as the pressure is increased. The reported results could be interpreted on the framework of itinerant magnetism and on the dependence of the magnetic structure on of the band structure. The suppression of magnetism seems to be related with a decrease of the Fe-Fe nearest neighbor distances that could promote a broadening of the $3 \mathrm{~d}$ band and hence a decrease of density of states at the Fermi level.
This work was supported by Spanish MICINN under MAT2009-14741-C02-01, MAT11/1077, ConsoliderIngenio in Molecular Nanoscience, CSD2007-00010

${ }^{1}$ H. Hasegawa and D. G. Pettifor, Phys. Rev. Lett. 50, 130-133 (1983).

${ }^{2}$ V. Iota, J. H. P. Klepeis, C. S. Yoo, J. Lang, D. Haskel, and G. Srajer, Appl. Phys. Lett. 90, 042505 (2007).

${ }^{3}$ I. A. Abrikosov, A. E. Kissavos, F. Liot, B. Alling, S. I. Simak, O. Peil, and A. V. Ruban, Phys. Rev. B 76, 014434 (2007) and references therein.

${ }^{4}$ P. Gorria, D. Martínez-Blanco, M. J. Pérez, J. A. Blanco, A. Hernando, M. A. Laguna-Marco, D. Haskel, N. Souza-Neto, R. I. Smith, W. G. Marshall, G. Garbarino, M. Mezouar, A. Fernández-Martínez, J. Chaboy, L. F. Barquín, J. A. R. Castrillón, M. Moldovan, J. I. G. Alonso, J. Zhang, A. Llobet, and J. S. Jiang, Phys. Rev. B 80, 064421 (2009).

${ }^{5}$ B. Rellinghaus, J. Kästner, Th. Schneider, and E. F. Wassermann, Phys. Rev. B 51, 2983-2993 (1995).

${ }^{6}$ P. Gorria, D. Martínez-Blanco, J. A. Blanco, A. Hernando, J. S. Garitaonandia, L. Fernández Barquín, J. Campo, and R. I. Smith, Phys. Rev. B 69, 214421 (2004).

${ }^{7}$ M. Eisenbach and G. M. Stocks, J. Appl. Phys. 105, 07E509 (2009).

${ }^{8}$ P. Crespo, M. Multigner, F. J. Castaño, R. Casero, A. Hernando, A. G. Escorial, L. Schultz, and S. N. Kaul, Phys. Rev. B 61, 14346-14349 (2000).

${ }^{9}$ J. M. Barandiarán, P. Gorria, I. Orúe, M. L. Fdez-Gubieda, F. Plazaola, and A. Hernando, Phys. Rev. B 54, 3026 (1996).

${ }^{10}$ A. Hernando, P. Crespo, F. J. Castaño, J. Arcas, M. Multigner, J. M. Barandiarán, and L. Fernández-Barquín, Phys. Rev. B 61, 3219-3222 (2000).

${ }^{11}$ M. van Schilfgaarde, I. A. Abrikosov, and B. Johansson, Nature (London) 400, 46-49 (1999).

${ }^{12}$ A. Martínez, J. Spottorno, A. I. Figueroa, F. Bartolomé, L. M. García, C. Prestipino, A. Hernando, and P. Crespo, Phys. Rev. B 82, 012406 (2010).

${ }^{13}$ H. K. Mao, J. Xu, and P. M. Bell, J. Geophys. Res. 91, 4673 (1986).

${ }^{14}$ F. Baudelet, S. Pascarelli, O. Mathon, J. P. Itié, A. Polian, M. d'Astuto, and J. C. Chervin, J. Phys.: Condens. Matter 17, S957 (2005).

${ }^{15}$ A. Martínez, J. J. Romero, A. F. Yang, G. R. Castro, V. G. Harris, J. C. Woicik, A. Hernando, and P. Crespo, IEEE Trans. Magn. 44(11), 3887-3890 (2008).

${ }^{16}$ P. Crespo, A. Hernando, R. Yavari, O. Drbohlav, A. García-Escorial, J. M. Barandiaran, and I. Oríe, Phys. Rev. B 48(10), 7134 (1993). 